This is the author's version of the accepted, peer-reviewed chapter:

Zlatar M., Brog JP., Tschannen A., Gruden-Pavlović M., Daul C. (2011) Density Functional Theory Study of the Multimode Jahn-Teller Effect - Ground State Distortion of Benzene Cation. In: Atanasov M., Daul C., Tregenna-Piggott P. (eds) Vibronic Interactions and the Jahn-Teller Effect. Progress in Theoretical Chemistry and Physics, vol 23. Springer, Dordrecht. Final version of the chapter is available online at:

https://doi.org/10.1007/978-94-007-2384-9 2 


\title{
Density Functional Theory Study of the Multimode Jahn-Teller Effect - Ground State Distortion of Benzene Cation
}

\author{
Matija Zlatar, Jean-Pierre Brog, Alain Tschannen, Maja Gruden-Pavlović and \\ Claude Daul
}

\begin{abstract}
The multideterminental-DFT approach performed to analyze Jahn-Teller (JT) active molecules is described. Extension of this method for the analysis of the adiabatic potential energy surfaces and the multimode JT effect is presented. Conceptually a simple model, based on the analogy between the JT distortion and reaction coordinates gives further information about microscopic origin of the JT effect. Within the harmonic approximation the JT distortion can be expressed as a linear combination of all totally symmetric normal modes in the low symmetry minimum energy conformation, which allows calculating the Intrinsic Distortion Path, IDP, exactly from the high symmetry nuclear configuration to the low symmetry energy minimum. It is possible to quantify the contribution of different normal modes to the distortion, their energy contribution to the total stabilization energy and how their contribution changes along the IDP. It is noteworthy that the results obtained by both multideterminental-DFT and IDP methods for different classes of JT active molecules are consistent and in agreement with available theoretical and experimental values. As an example, detailed description of the ground state distortion of benzene cation is given.
\end{abstract}

\footnotetext{
Matija Zlatar

Department of Chemistry, University of Fribourg, Fribourg, Switzerland; Center for Chemistry, IHTM, University of Belgrade, Belgrade, Serbia, e-mail: matijaz@chem.bg.ac.rs

Maja Gruden-Pavlović

Faculty of Chemistry, University of Belgrade, Belgrade, Serbia

e-mail: gmaja@chem.bg.ac.rs

Claude Daul

Department of Chemistry, University of Fribourg, Fribourg, Switzerland e-mail: claude.daul@unifr.ch
} 


\section{Introduction}

In contempt of the great progress and development of various experimental techniques for studying the Jahn-Teller (JT) effect [1, 2], computational methods are necessary to understand the microscopic origin and to get deeper insight into the vibronic coupling effects. Traditional computational methods, can still be used even where non-adiabatic effects are important, if a perturbation approach is introduced to the Born-Oppenheimer approximation. This is possible if the adiabatic potential energy surface can be accurately determined, as in the case of Density Functional Theory (DFT). In this way all the standard concepts in theoretical chemistry are still useful for the elucidation and prediction of the properties of JT active molecules and many manifestations of the JT effect can be understood within the Born-Oppenheimer approximation. DFT is today widely used method in quantum chemistry and allows obtaining accurate results at low computational cost. Moreover, it helps to understand chemical origin of the effect under study. Contrary to some beliefs that DFT is not adequate for degenerate states [3, 4], DFT can be applied to both degenerate and excited states, as formally proved by the reformulation of the original Hohenberg-Kohn theorems, i.e. constrained search method and finite temperature DFT [5]. Furthermore, Kohn-Sham (KS) DFT, as the most common practical way of using DFT, is based on the equations equivalent and fully compatible with the equations used in the wave-function based methods. However, precaution is necessary, conventional DFT cannot be used as a black-box, e.g. it needs to be extended to treat a multiplet problem [6, 7, 8]. KS-DFT in its present implementations is not able to derive correct energies in the case of orbital degeneracy $[3,9,10]$. On the other hand, multideterminental-DFT approach, developed by Daul et al. [11, 12], can be successfully applied for detailed analysis of the JT active molecules $[10,11,12,13,14,15,16,17,18,19,20]$. This method gives the JT parameters, Fig. 1, as well as corresponding geometries with good accuracy.

The set of parameters depicted on the Fig. 1 characterizes the potential energy surface of the JT active molecules. The distortion from a high symmetry (HS) nuclear arrangement, due to the JT effect, towards a lower symmetry (LS) energy minimum conformation, is a linear displacement on the $3 N-6$ potential energy surface. In the ideal case, the distortion would correspond to the movements of nuclei along one normal mode that belongs to a non-totally symmetric irreducible representation of the HS point group of a molecule. Nevertheless, this can be true only for simple molecules. In complex molecules, the JT distortion is a superposition of many different normal coordinates. The appraising of the influence of different normal modes on the JT effect is referred as a multimode problem. Recently, we have proposed to express the JT distortion as a linear combination of all totally symmetric normal modes in the LS minimum energy conformation [12]. Our approach to the multimode JT problem is an alternative to the method based on the transformations in the coordinate system which reduces multimode to one-mode problem [2, 21]. Recently, Bersuker et al. used the method of coordinate transformation of the HS symmetrized displacements of $\mathrm{CO}_{3}$ to reduce the two-mode to one-mode problem [22]. The normal coordinates in the LS are not identical with the normal coordi- 


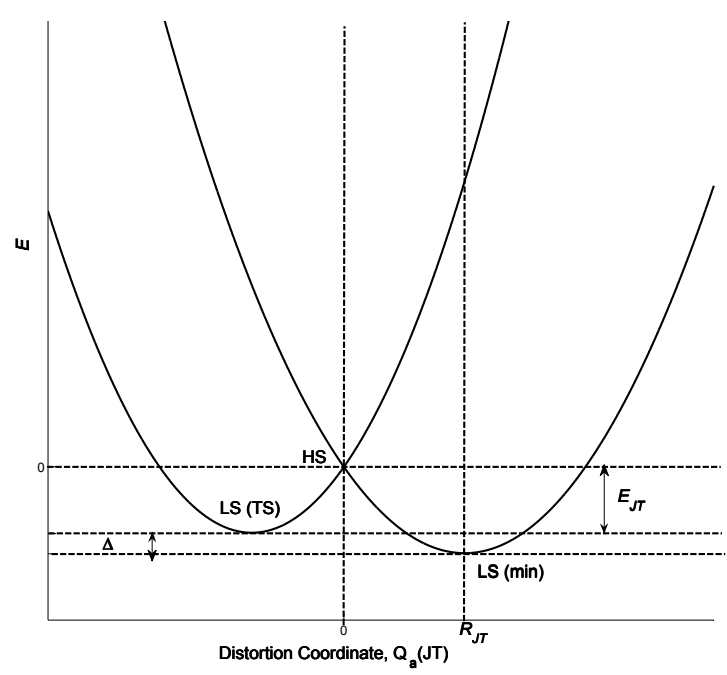

Fig. 1 Qualitative cross section through the potential energy surface, along JT active distortion $\mathbf{Q}_{a}$; Definition of the JT parameters-the JT stabilization energy, $E_{\mathrm{JT}}$, the warping barrier, $\Delta$, the $\mathrm{JT}$ radius, $R_{\mathrm{JT}}$

nates in the HS, so the idea of using LS structure as a reference point is in contrast with usual treatment of the JT effect which starts from the HS configuration. Still, we can easily correlate the normal modes of the LS structure to the HS ones, using the method of Hug [23], thus having the connection with the common exploration of the JT effect. Our model is based on the symmetry rules developed by Bader $[24,25,26]$ and Pearson $[27,28]$ for chemical reactions. Any displacement on the potential energy surface has to be totally symmetric and consequently a superposition of the totally symmetric normal coordinates. Within harmonic approximation the potential energy surface has a simple analytical form, hence with our model it is possible to directly separate contributions of different normal modes to the JT distortion, $\mathbf{R}_{\mathrm{JT}}$, their energy contribution to the $E_{\mathrm{JT}}$ and the forces at the HS point. This allows calculating the path of minimal energy, Intrinsic Distortion Path (IDP), exactly from the HS point to the LS energy minimum. Inspection of the IDP gives additional information about microscopic origin and mechanism of the distortion.

\section{Computational Details}

The DFT calculations reported in this work have been carried out using the Amsterdam Density Functional program package, ADF2009.01 [29, 30, 31]. The local density approximation (LDA) characterized by the Vosko- Willk-Nusair (VWN) [32] parametrization have been used for the geometry optimizations. An all electron Triple-zeta Slater-type orbitals (STO) plus one polarization function (TZP) basis set 
has been used for all atoms. All calculations were spin-unrestricted. Separation of orbital and geometrical symmetry, as used in the calculation of the energies of the HS nuclear configurations, is done using SYMROT subblock in the QUILD program, version 2009.01 [33], provided in the ADF2009.01 program package. Analytical harmonic frequencies were calculated [34, 35], and were analyzed with the aid of PyVib2 1.1 [36]. Vibrations are illustrated using the vibrational energy distribution representation [23]. The different colours indicate the direction of the displacement vector, while the volumes of the spheres are proportional to the contribution made by the individual nuclei to the energy of the vibrational mode.

\section{Methodology}

\subsection{DFT for the Calculation of the Jahn-Teller Parameters}

In order to get the JT parameters $\left(E_{\mathrm{JT}}, \Delta, R_{\mathrm{JT}}\right)$, Fig. 1 , it is necessary to know the energies and geometries of the HS and LS nuclear arrangements. For the LS structures, as they are in non-degenerate electronic states this is straightforward. A geometry optimization, constraining the structure to the LS point group, with proper orbital occupancy will yield the different LS geometries and energies, that correspond to the minimum and to the transition state on the potential energy surface.

Electronic structure of the HS point, on the other hand, must be represented with at least two Slater determinants, consequently, using a single determinant DFT is troublesome. In a non-empirical approach to calculate the JT distortion using DFT [11] it was proposed to use the average of configuration (AOC) type calculation to generate the electron density. This is a SCF calculation where the electrons of degenerate orbitals are distributed equally over the components of the degenerate irreps leading to a homogeneous distribution of electrons with partial occupation. In this way, the $A_{1}$ symmetry of the total density in the HS point group is retained. E.g. for $e^{1}$ configuration this will mean to place 0.5 electrons into each of the two $e$ orbitals. This calculation yields the geometry of the high symmetry species. Although, AOC calculation gives us geometry of a HS point, one needs to be cautious with using simply this energy. Electronic distribution with an electron (or a hole) evenly distributed between the degenerate orbitals leads to a lower energy than in the case of an integer orbital occupancy. Practical solution to the problem is to perform a single point calculation imposing the HS symmetry on the nuclear geometry and the LS symmetry of the electron density. This gives the energy of a Slater determinant with an integer electron occupation of the molecular orbitals. To obtain the energies of the degenerate states at HS point, it is necessary to evaluate the energies of all possible single determinants with integer electron occupations.

To summarize: the difference between the energy obtained by the single point calculations on the HS nuclear geometry with the LS electron density, and the energy obtained by geometry optimization of the LS structure with the same electron 
distribution is $E_{\mathrm{JT}} . \Delta$ is the difference in energy between the two LS structures with different electron distributions. The JT radius, $R_{\mathrm{JT}}$ is given by the length of the distortion vector $\left(\mathbf{R}_{\mathrm{JT}}\right)$ between the HS configuration $\left(\mathbf{R}_{\mathrm{HS}}\right)$ and the LS stationary points $\left(\mathbf{R}_{\mathrm{LS}}\right)$.

$$
\mathbf{R}_{\mathrm{JT}}=\mathbf{R}_{\mathrm{HS}}-\mathbf{R}_{\mathrm{LS}}=R_{\mathrm{JT}} \mathbf{u}
$$

This computational procedure, for the particular example of the ground state JT effect in benzene cation, is outlined in the Fig. 2 .

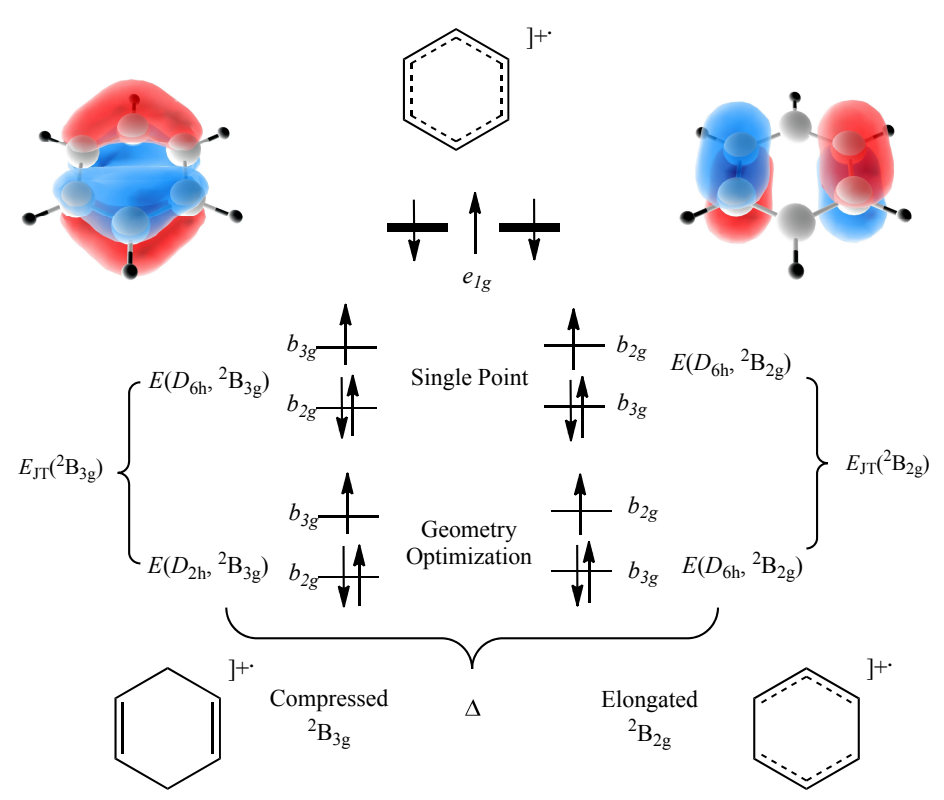

Fig. 2 Multideterminental-DFT approach for the calculation of the JT parameters for the ground state distortion of $\mathrm{C}_{6} \mathrm{H}_{6}^{+}$

\subsection{Analysis of the multimode Jahn-Teller effect}

Geometry of the LS energy minimum conformation is chosen to be the origin of the configuration space, $\mathbf{R}_{\mathrm{LS}}=\mathbf{0}$. Every point on the potential energy surface can be represented by a $3 N$ dimensional vector $\mathbf{R}_{\mathrm{X}}$ using mass-weighted generalized coordinates relative to the origin. Within the harmonic approximation, it is possible 
to express $\mathbf{R}_{\mathrm{X}}$ as a linear combination of all totally symmetric normal coordinates $\left(N_{a_{1}}\right)$ from the LS energy minimum conformation:

$$
\mathbf{R}_{\mathrm{X}}=\mathbb{Q} \mathbf{w}_{\mathrm{X}}
$$

$\mathbb{Q}$ is the $3 N \times N_{a_{1}}$ matrix with a columns being mass-weighted totally symmetric normal coordinates, obtained by the DFT calculations in the LS minimum energy conformation. $\mathbf{w}_{\mathrm{X}}$ is the $N_{a_{1}}$ dimensional vector containing the weighting factors, $w_{\mathrm{HSk}}$, which can be easily obtained solving the linear problem, eq. 2 . $w_{\mathrm{HSk}} \mathrm{S}$ represent the contribution of the displacements along the different totally symmetric normal coordinates to the $\mathbf{R}_{\mathrm{X}}$. The energy of the nuclear configuration $\mathbf{R}_{\mathrm{X}}, E_{\mathrm{X}}$, relative to the energy of the origin, in harmonic approximation, is expressed as the sum of the energy contributions of all $N_{a_{1}}$ LS totally symmetric normal coordinates:

$$
E_{\mathrm{X}}=\sum_{\mathrm{k}=1}^{N_{a_{1}}} E_{\mathrm{kX}}=\frac{1}{2} \sum_{\mathrm{k}=1}^{N_{a_{1}}} w_{\mathrm{Xk}}^{2} \mathbf{Q}_{\mathrm{k}}^{2} \lambda_{\mathrm{k}}
$$

where $\lambda_{\mathrm{k}}$ are the eigenvalues of the Hessian from the DFT calculations in the LS minimum energy conformation. Using these considerations we can analyze the multimode JT problem by expressing the $\mathbf{R}_{\mathrm{JT}}$ as a superposition of all the LS totally symmetric normal coordinates, and directly obtaining the energy contributions of all the normal modes to the total stabilization energy:

$$
\begin{gathered}
\mathbf{R}_{\mathrm{JT}}=\mathbb{Q} \mathbf{w}_{\mathrm{kJT}} \\
E_{\mathrm{JT}}=\sum_{\mathrm{k}=1}^{N_{a_{1}}} E_{\mathrm{kJT}}=\frac{1}{2} \sum_{\mathrm{k}=1}^{N_{a_{1}}} w_{\mathrm{kJT}}^{2} \mathbf{Q}_{\mathrm{k}}^{2} \lambda_{\mathrm{k}}
\end{gathered}
$$

The vector $\mathbf{R}_{\mathrm{JT}}=\mathbf{R}_{\mathrm{HS}}$ defines the straight path from the HS point to the LS minimum - direct path. Direct path is equivalent to the interaction mode of Bersuker et al. [2, 21, 22]. It contains essential information on the vibronic coupling at the HS point. Direct path is in general different from the minimal energy path from HS point on the potential energy surface to LS global minimum. The force along the normal mode $\mathbf{Q}_{\mathrm{k}}, \mathbf{F}_{\mathrm{Xk}}$, which drives the nuclei along that coordinate to the minimum, at any point $\mathbf{R}_{\mathrm{X}}$ is defined as a derivative of the energy over the Cartesian coordinates. In the HS point this will lead information about the main driving force for the JT distortion from the HS to the LS. The total distortion force, $\mathbf{F}_{\mathrm{Xtot}}$, is given as a vector sum of the individual forces. $\mathbf{F}_{\text {Xtot }}$ gives the direction from one to the another point on the adiabatic potential energy surface in a way of maximizing the decrease of energy.

$$
\mathbf{F}_{\mathrm{Xtot}}=\sum_{\mathrm{k}=1}^{N_{a_{1}}} \mathbf{F}_{\mathrm{Xk}}=\frac{1}{2} \sum_{\mathrm{k}=1}^{N_{a_{1}}} w_{\mathrm{Xk}} \lambda_{\mathrm{k}} \mathbb{M}^{1 / 2} \mathbf{Q}_{\mathrm{k}}
$$

$\mathbb{M}$ is a diagonal $3 N \times 3 N$ matrix with atomic masses in triplicates as elements $\left(m_{1}, m_{1}, m_{1}, m_{2}, \ldots, m_{N}\right)$. 
The simple analytical form of the potential energy surface in the harmonic approximation allows calculating the minimal energy path, IDP, exactly from the HS to the LS energy minimum. Along the IDP path the contributions of the different modes to the distortion will change, contrary to the direct path. At the HS only JT active modes contribute. After the first step, the symmetry is lowered and the other modes will mix in. Analysis of the IDP allows getting very detailed picture on the interaction between the deformation of the electron distribution and the displacements of the nuclei, not accessible through an experiment. We should make the remark that the $E_{\mathrm{JT}}$ obtained with the IDP method is a complementary to the method based on the multideterminental-DFT described in previous section. For the IDP analysis one needs information about HS and LS geometries and LS normal modes, thus, avoiding the problems of evaluation of the energies of the degenerate states. Another important thing is that this model is completely theoretical, without additional fitting, all the vibrations that can contribute to the distortion are considered, and all the equations can be solved analytically in the harmonic approximation.

\section{Results and Discussion}

Using the multideterminental-DFT procedure in the conjunction with the IDP method we have analyzed a number of different JT active molecules, Table 1. The considered molecules, in HS nuclear configuration, have a doubly degenerate electronic ground state which is coupled with a doubly degenerate vibrations. Thus, they belong to the $E \otimes e \mathrm{JT}$ systems. Apart from that, these systems differ in the nature of chemical bonding, symmetry of the distortion, range of the $E_{\mathrm{JT}}, \Delta$ and $R_{\mathrm{JT}}$, the number of atoms, and hence the number of different normal modes that need to be considered in the IDP analysis.

Table 1 Results of multideterminental-DFT calculations and IDP method performed to analyze the JT effect of selected compounds; energies are given in $10^{3} \mathrm{~cm}^{-1} ; R_{\mathrm{JT}}$ in $(\mathrm{amu})^{1 / 2} \AA ; N$ is the number of atoms in a molecule; $N_{a_{1}}$ is the number of totally symmetrical vibrations in LS minimum

\begin{tabular}{|c|c|c|c|c|c|c|c|c|}
\hline & Distortion & $E_{\mathrm{JT}}(\mathrm{DFT})$ & $E_{\mathrm{JT}}(\mathrm{IDP})$ & $E_{\mathrm{JT}}(\exp )$ & $\Delta$ & $R_{\mathrm{JT}}$ & $3 N-6$ & $N_{a_{1}}$ \\
\hline $\mathrm{Cu}_{3}$ & $D_{3 h} \longrightarrow C_{2 v}$ & $0.53[18]$ & 0.37 & $0.28-0$ & 0.12 & 1.12 & 3 & 2 \\
\hline $\mathrm{VCl}_{4}$ & $T_{d} \longrightarrow D_{2 d}$ & $0.04[11,12]$ & 0.01 & $0.03-0.08[37,38,3$ & 0.01 & 0.10 & 9 & 2 \\
\hline $\mathrm{C}_{5} \mathrm{H}_{5}^{-}$ & $D_{5 h} \longrightarrow C_{2 v}$ & $1.25[12]$ & 1.24 & $1.24[41]$ & 0.00 & 0.17 & 24 & 9 \\
\hline $\mathrm{CoCp}_{2}$ & $D_{5 h} \longrightarrow C_{2 v}$ & $0.81[16,18]$ & 0.80 & $0.15-1.05[42]$ & 0.00 & 0.35 & 57 & 16 \\
\hline $\mathrm{MnCp}_{2}$ & $D_{5 h} \longrightarrow C_{2 v}$ & $0.71[18]$ & 0.72 & $\sim 0.35[42,43]$ & 0.00 & 0.77 & 57 & 16 \\
\hline $\mathrm{Cu}(\mathrm{en})_{3}^{2+}$ & $D_{3} \longrightarrow C_{2}$ & $2.12[19]$ & 2.70 & $2.00 \pm 0.20[44]$ & 0.57 & 2.34 & 105 & 53 \\
\hline $\mathrm{Cu}(\mathrm{eg})_{3}^{2+}$ & $D_{3} \longrightarrow C_{2}$ & $2.31[19]$ & 2.50 & n.r. & 1.41 & 3.36 & 87 & 44 \\
\hline
\end{tabular}

Without going into details, because some of these results have already been published $[11,12,16,18,19,20]$, we want to point out that the results obtained by 
multideterminental-DFT and IDP methods are consistent, and in a good agreement with experimental data and various theoretical methods, Table 1 . In all cases we were able to rationalize which are the totally symmetric normal modes of the LS structure that contribute to the JT distortion at the HS point, their energy contribution to the $E_{\mathrm{JT}}$, and how their contribution changes along the IDP, even in the case when we have to deal with 53 totally symmetric normal modes in tris(ethylenediamine) $\mathrm{Cu}$ (II) [19]. In the beginning, the JT active modes, that are the basis of the nontotally symmetric irreps in HS point group, are dominating. If there are several appropriate vibrations, harder ones will be dominant in the first step, while the softer ones take over along the IDP. The contribution of the other modes is becoming more important for the direction of the relaxation with increasing deviation from the HS geometry. Their contribution to the stabilization energy however is minor.

As an example, results for benzene cation are presented in the following section, which allows the reader to understand the concepts behind both the multideterminentalDFT and IDP methods.

\subsection{Multimode Jahn-Teller effect in the Ground State of Benzene Cation}

Benzene cation has a ${ }^{2} E_{1 g}$ ground electronic state in regular hexagonal nuclear configuration, $D_{6 h}$ point group, with a three electrons (one hole) in the doubly degenerate orbital. Hence, benzene cation is JT active and prefers a conformation of lower symmetry. According to group theory, the distortion coordinate is $e_{2 g}$ $\left(E_{1 g} \times E_{1 g} \subset A_{1 g}+\left[A_{2 g}\right]+E_{2 g}\right)$. Descent in symmetry goes to $D_{2 h}$. In $D_{2 h}$ electronic state splits into in ${ }^{2} B_{2 g}$ and ${ }^{2} B_{3 g}$. Qualitatively, direction of the distortion can be understood by looking to the frontier orbitals of the cation, Fig. 2. The direction of distortion is in the way of maximizing the bonding interactions and minimizing antibonding interactions in the doubly occupied molecular orbital originated from the $e_{1 g}$ orbital in $D_{6 h}$. The ${ }^{2} B_{2 g}$ state corresponds to the elongated (acute) geometry, with two $\mathrm{C}-\mathrm{C}$ bonds longer and four other shorter. According to our DFT calculations it is the global minimum on the potential energy surface. The ${ }^{2} B_{3 g}$ electronic state corresponds to the compressed (obtuse) geometry, with two shorter and four longer $\mathrm{C}-\mathrm{C}$ bonds. This structure is a transition state for the process of the pseudorotation along the lowest sheet of potential energy surface. JT effect in this system is is summarized in the Fig. 2, while the results of the DFT calculations are presented in the Table 2. According to our DFT calculations $E_{\mathrm{JT}}=880 \mathrm{~cm}^{-1}$ for this system. The elongated structure is found to be more stable than the compressed one for $\Delta=32 \mathrm{~cm}^{-1}$. IDP method gives $E_{\mathrm{JT}}$ in a good agreement with the DFT calculations, with the smaller values for around $40 \mathrm{~cm}^{-1} . \Delta$ value from IDP method can be estimated from a difference between the two $E_{\mathrm{JT}}$ for two electronic states and is $48 \mathrm{~cm}^{-1}$, a slightly higher value than obtained with multideterminental-DFT. Small energy difference between the ${ }^{2} B_{2 g}$ and ${ }^{2} B_{3 g}$ states suggests that the second 
order JT effects and anharmonicity are small and that JT effect in benzene cation is dynamic.

Table 2 Results of the DFT calculations performed to analyze the JT effect in $\mathrm{C}_{6} \mathrm{H}_{6}^{+}$; energies (LDA) are given in $\mathrm{eV}$; the JT parameters $E_{\mathrm{JT}}$ and $\Delta$ are given in $\mathrm{cm}^{-1}$ and $R_{\mathrm{JT}}$ in $(\mathrm{amu})^{1 / 2} \AA$

\begin{tabular}{cccc}
\hline \hline Occupation State Geometry & Energy \\
\hline$e_{1 g}^{0.75} e_{1 g}^{0.75}$ & ${ }^{2} E_{1 g}$ & $D_{6 h}$ & -70.9461 \\
$b_{3 g}^{2} b_{2 g}^{1}$ & ${ }^{2} B_{2 g}$ & $D_{6 h}$ & -70.9122 \\
$b_{2 g}^{2} b_{3 g}^{1}$ & ${ }^{2} B_{3 g}$ & $D_{6 h}$ & -70.9141 \\
$b_{3 g}^{2} b_{2 g}^{1}$ & ${ }^{2} B_{2 g}$ & $D_{2 h}$ & -71.0212 \\
$b_{2 g}^{2} b_{3 g}^{1}$ & ${ }^{2} B_{3 g}$ & $D_{2 h}$ & -71.0172 \\
\hline$E_{\mathrm{JT}}$ & ${ }^{2} B_{2 g}$ & & 879.2 \\
$E_{\mathrm{JT}}$ & ${ }^{2} B_{3 g}$ & & 831.6 \\
$\Delta$ & & & 32.2 \\
$R_{\mathrm{JT}}$ & ${ }^{2} B_{2 g}$ & & 0.09 \\
$R_{\mathrm{JT}}$ & ${ }^{2} B_{3 g}$ & & 0.09 \\
\hline$E_{\mathrm{JT}}(\mathrm{IDP})$ & ${ }^{2} B_{2 g}$ & & 839.1 \\
$E_{\mathrm{JT}}(\mathrm{IDP})$ & ${ }^{2} B_{3 g}$ & & 791.1 \\
\hline \hline
\end{tabular}

Benzene cation has been thoroughly studied over the years [45, 46, 47, 48, 49, $50,51,52,53,54,55]$. The values of the JT parameters largely depend on the used method and model employed. Calculations based on the Hartree-Fock and MP2 methods, not surprisingly, overestimate the $E_{\mathrm{JT}}$ [45]. Experimental methods and models that consider only one mode, on the other hand, are underestimating $E_{\mathrm{JT}}$, e.g. experimental value of Lindner et al is $266 \mathrm{~cm}^{-1}$ [46]. Recent calculations based on complete active space or DFT, including this study, are much more consistent and in general agreement that $E_{\mathrm{JT}}$ falls in range between 700 and $1000 \mathrm{~cm}^{-1}[48,53,54]$. Even more inconsistency is found for the value of $\Delta$, and alternately both states are reported to be a global minimum $[47,48]$. This is due to the small value of $\Delta$ which is in the range of errors of the calculations. Experimentally $\Delta$ is estimated to be 8 $\mathrm{cm}^{-1}[46]$ and the elongated form to be a minimum. Independently of the particular value, the $\Delta$ is rather small, smaller than zero point vibrational energy, and JT effect is dynamic. Köppel et al [49, 50, 51, 52] and Sardar et al [55] did detailed dynamic study of the multistate multimode problem in benzene cation.

Benzene cation in $D_{6 h}$ symmetry has four $e_{2 g}$ and two $a_{1 g}$ vibrations. After descent in symmetry to $D_{2 h}$ they all become totally symmetric (one component of each pair in the case of the degenerate vibrations). Thus, in $D_{2 h}$ symmetry there are six totally symmetric normal modes, which can mix and all of them can contribute to the distortion. Out of six totally symmetric normal modes in $D_{2 h}$, with our model we are able to identify the three most important vibrations contributing to the JT distortion: $\mathrm{C}-\mathrm{C}-\mathrm{C}$ bend $\left(591 \mathrm{~cm}^{-1}\right), \mathrm{C}-\mathrm{C}-\mathrm{H}$ bend $\left(1166 \mathrm{~cm}^{-1}\right)$ and $\mathrm{C}-\mathrm{C}$ stretch $\left(1556 \mathrm{~cm}^{-1}\right)$. This is in agreement with previous studies [48, 53, 54]. These three vibrations are illustrated on the Fig. 3 using the vibrational energy distribu- 


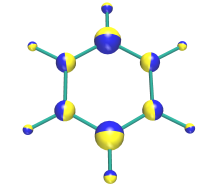

(a) $591 \mathrm{~cm}^{-1}(\mathrm{C}-\mathrm{C}-\mathrm{C}$ bend $)$

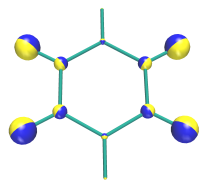

(b) $1166 \mathrm{~cm}^{-1}(\mathrm{C}-\mathrm{C}-\mathrm{H}$ bend $)$

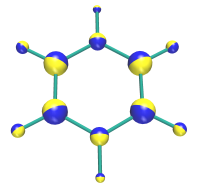

(c) $1556 \mathrm{~cm}^{-1}(\mathrm{C}-\mathrm{C}$ stretch $)$

Fig. 3 Vibrational energy distribution representation of the three most important $a_{1} g$ vibrations in $D_{2 h}$ symmetry of $\mathrm{C}_{6} \mathrm{H}_{6}^{+}$, corresponding to the three $e_{2 g} \mathrm{JT}$ active vibrations in $D_{6 h}$ symmetry. The different co lours indicate the direction of the displacement vector; the volume of the spheres is proportional to the contribution made by the individual nuclei to the energy of the vibrational mode

tion representation [23]. These three vibrations corresponds to $e_{2 g}$ vibrations in $D_{6 h}$ configuration of benzene cation, and contribute $99 \%$ to the $R_{\mathrm{JT}}$ and $98 \%$ to the $E_{\mathrm{JT}}$, Fig. 4.

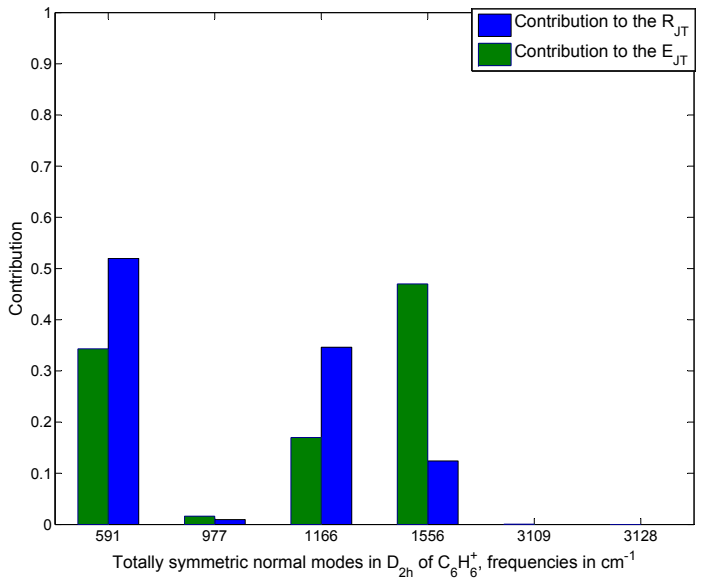

Fig. 4 Contribution of the $6 a_{1 g}$ normal modes in $D_{2 h}$ to the $E_{\mathrm{JT}}$ (green) and to the $R_{\mathrm{JT}}$ (blue) of $\mathrm{C}_{6} \mathrm{H}_{6}^{+}$ 

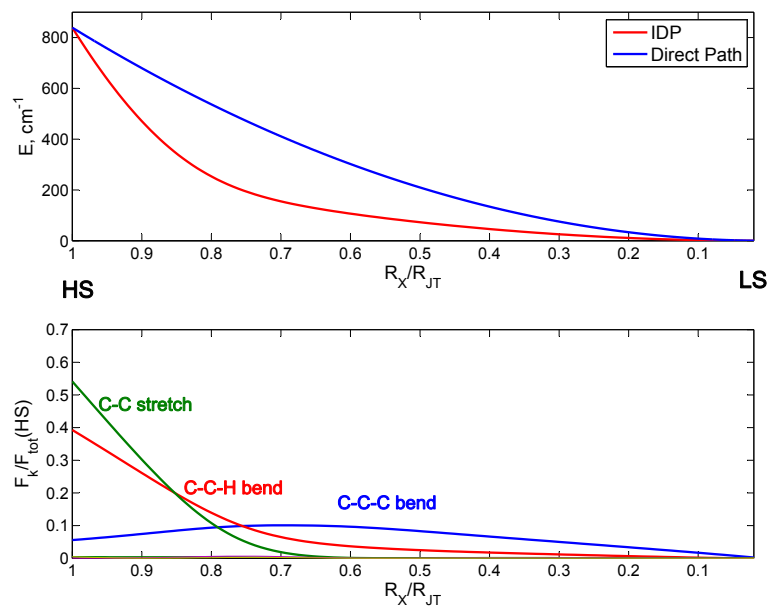

Fig. 5 Difference between the direct Path and IDP (up) and changes of the forces (normalized to the total distortion force at HS point) of the $6 a_{1 g}$ normal modes in $D_{2 h}$, along the IDP of $\mathrm{C}_{6} \mathrm{H}_{6}^{+}$

IDP method gives further insight into the vibronic coupling in benzene cation. Figure 5. shows the energy differences between the IDP and the direct path and the changes of the forces of the different normal modes along the IDP. On the potential energy profile it is possible to distinguish two distinct regions. In the first region energy is changing faster. After $25 \%$ of the path, already c.a. $75 \%$ of the $E_{\mathrm{JT}}$ is obtained. In this region all three dominant vibrations are contributing, with the hardest of the three, $\mathrm{C}-\mathrm{C}$ stretch being most important. Contribution of the $\mathrm{C}-\mathrm{C}$ stretch becomes minor in the second region. In the second region the change of the energy is small and potential energy surface is flat. In this region, the molecule, after achieving most of the stabilization energy due to the JT effect, relaxes towards the global minimum. The softest mode, $\mathrm{C}-\mathrm{C}-\mathrm{C}$ bend is the most important in this second region.

\section{Conclusions}

In this paper multideterminental-DFT method for the qualitative and quantitative analysis of the adiabatic potential energy surfaces of the JT active molecules is presented. It is shown how DFT can be successfully applied for the calculation of the JT parameters. In addition, the analysis of the multimode JT effect using the IDP method is shown. The essence of this model is to express the JT distortion as a linear combination of all totally symmetric normal modes in the LS minimum energy 
conformation. This is an alternative to the method that starts from the HS structure and reduces the multimode problem to a single interaction mode [2, 21, 22]. So far, IDP method has not been explored in the cases of the hidden JT effect, or where the influence of the pseudo JT effect is important, and combination of two different approaches may further develop this topic. The IDP analysis answers the questions which are the totally symmetric normal modes of the LS structure that contribute to the JT distortion at the HS point, how they contribute to the $E_{\mathrm{JT}}$, and how their contributions change along the IDP. We want to point out that this reduction of the multimode problem to the IDP solves the structural part of the problem, but not the dynamical part, meaning the changes in vibrational frequencies by the multimode distortion.

The performance of the multideterminental-DFT and the IDP model has been evaluated for different JT active molecules. In all cases the results obtained by both methods are consistent and in the excellent agreement with experimental and theoretical values reported in the literature. The detailed description of the multimode JT distortion of the ground state of benzene cation is shown as an example of utility of both methods. Both schemes are fast and accurate and can be considered as re-

liable tools for the investigation of adiabatic potential energy surfaces of JT active molecules and for a better understanding of the JT effect.

Acknowledgements This work was supported by the Swiss National Science Foundation and the Serbian Ministry of Science (Grant no. 172035). The authors would like to thank to Professor Carl-Wilhelm Schläpfer for the constant interest and advice.

\section{References}

[1] H.A. Jahn, E. Teller, Proc. R. Soc. London, Ser. A 161, 220 (1937)

[2] I.B. Bersuker, The Jahn-Teller Effect (Cambridge University Press, 2006)

[3] I.B. Bersuker, J. Comp Chem. 18(2), 260 (1997)

[4] I.G. Kaplan, J. Mol. Struct. 838, 39 (2007)

[5] R.G. Parr, W. Yang, Density-Functional Theory of Atoms and Molecules (Oxford University Press, 1989)

[6] T. Ziegler, A. Rauk, E.J. Baerends, Theor. Chim. Acta. 43, 261 (1977)

[7] C. Daul, Int. J. Quant. Chem. 52, 867 (1994)

[8] C.A. Daul, K. Doclo, C.A. Stückl, in Recent Advances in Density Functional Methods, Part II, ed. by D.P. Chong (World Scientific Publishing Company, 1997)

[9] M. Atanasov, C. Daul, Chimia 59, 504 (2005)

[10] D. Reinen, M. Atanasov, W. Massa, Z. Anorg. Allg. Chem. 632, 1375 (2006)

[11] R. Bruyndonckx, C. Daul, P.T. Manoharan, E. Deiss, Inorg. Chem. 36, 4251 (1997) 
[12] M. Zlatar, C.W. Schläpfer, C. Daul, in The Jahn-Teller-Effect Fundamentals and Implications for Physics and Chemistry, ed. by H. Koeppel, D.R. Yarkoni, H. Barentzen (Springer Series in Chemical Physics, 97, 2009), pp. 131-165

[13] T.K. Kundu, R. Bruyndonckx, C. Daul, P.T. Manoharan, Inorg. Chem. 38, 3931 (1999)

[14] M. Atanasov, P. Comba, J. Mol. Struct. 38, 157 (2007)

[15] M. Atanasov, P. Comba, C.A. Daul, A. Hauser, J. Phys. Chem. A 38, 9145 (2007)

[16] M. Zlatar, C.W. Schläpfer, E.P. Fowe, C. Daul, Pure Appl. Chem. (2009)

[17] D. Reinen, M. Atanasov, P. Köhler, D. Babel, Coordination Chemistry Reviews 254, 2703 (2010)

[18] M. Zlatar, M. Gruden-Pavlović, C.W. Schläpfer, C. Daul, Journal of Molecular Structure: THEOCHEM 954, 86 (2010)

[19] M. Gruden-Pavlović, M. Zlatar, , C.W. Schläpfer, C. Daul, Journal of Molecular Structure: THEOCHEM 954, 80 (2010)

[20] M. Zlatar, M. Gruden-Pavlović, C.W. Schläpfer, C. Daul, Chimia 64, 161 (2010)

[21] I.B. Bersuker, V.Z. Polinger, Vibronic interactions in Molecules and Crystals (Springer-Verlag: Berlin, 1989)

[22] Y. Liu, I.B. Bersuker, W. Zou, J.E. Boggs, J. Chem. Theory Comput. 5, 2679 (2009)

[23] W. Hug, M. Fedorovsky, Theor. Chem. Acc. 119, 113 (2008)

[24] R.F.W. Bader, Mol. Phys. 3, 137 (1960)

[25] R.F.W. Bader, Can. J. Chem. 40, 1164 (1962)

[26] R.F.W. Bader, A.D. Bandrauk, J. Chem. Phys. 49, 1666 (1968)

[27] R.G. Pearson, J. Am. Chem. Soc. 91(18), 4947 (1969)

[28] R.G. Pearson, Symmetry Rules for Chemical reactions (A Willey-Interscience Publication, 1976)

[29] E.J. Baerends, J. Autschbach, D. Bashford, A. Bérces, F.M. Bickelhaupt, C. Bo, P.M. Boerrigter, L. Cavallo, D.P. Chong, L. Deng, R.M. Dickson, D.E. Ellis, M. van Faassen, L. Fan, T.H. Fischer, C.F. Guerra, A. Ghysels, A. Giammona, S. van Gisbergen, A. Götz, J. Groeneveld, O. Gritsenko, M. Grning, F. Harris, P. van den Hoek, C. Jacob, H. Jacobsen, L. Jensen, G. van Kessel, F. Kootstra, M.V. Krykunov, E. van Lenthe, D.A. McCormack, A. Michalak, M. Mitoraj, J. Neugebauer, V.P. Nicu, L. Noodleman, V.P. Osinga, S. Patchkovskii, P.H.T. Philipsen, D. Post, C.C. Pye, W. Ravenek, J.I. Rodrguez, P. Ros, P.R.T. Schipper, G. Schreckenbach, M. Seth, J.G. Snijders, M. Sola, M. Swart, D. Swerhone, G. te Velde, P. Vernooijs, L. Versluis, L. Visscher, O. Visser, F. Wang, T.A. Wesolowski, E.M. van Wezenbeek, G. Wiesenekker, S.K. Wolff, T.K. Woo, A.L. Yakovlev, T. Ziegler. Adf2009.01. http://www.scm.com (2009)

[30] C.F. Guerra, J.G. Snijders, G. te Velde, E.J. Baerends, Theor. Chem. Acc. 99, 391 (1998)

[31] G. te Velde, F.M. Bickelhaupt, S.J.A. van Gisbergen, C.F. Guerra, E.J. Baerends, J.G. Snijders, T. Ziegler, J. Comput. Chem. 22, 931 (2001) 
[32] S. Vosko, L. Wilk, M. Nusair, Can. J. Phys. 58, 1200 (1980)

[33] M. Swart, F.M. Bickelhaupt, J. Comp. Chem. 29, 724 (2007)

[34] A. Bérces, R.M. Dickson, L. Fan, H. Jacobsen, D. Swerhone, T. Ziegler, Comput. Phys. Commun. 100, 247 (1997)

[35] H. Jacobsen, A. Bérces, D. Swerhone, T. Ziegler, Comput. Phys. Commun. 100, 263 (1997)

[36] M. Fedorovsky. Pyvib2, a program for analyzing vibrational motion and vibrational spectra. http://pyvib2.sourceforge.net (2007)

[37] F.A. Blankenship, R.L. Belford, J. Chem. Phys. 36, 633 (1962)

[38] Y. Morino, H. Uehara, J. Chem. Phys. 45, 4543 (1966)

[39] R.B. Johannesen, G.A. Candela, T. Tsang, J. Chem. Phys. 48, 5544 (1968)

[40] J.H. Ammeter, L. Zoller, J. Bachmann, P. Baltzer, E., R. Bucher, E. Deiss, Helv. Chim. Acta 64, 1063 (1981)

[41] B.E. Applegate, J. Bezant, T.A. Miller, J. Chem. Phys. 114, 4869 (2001)

[42] R. Bucher, Esr-untersuchungen an jahn-teller-aktiven sandwitchkomplexen. Ph.D. thesis, ETH Zürich (1977)

[43] J.H. Ammeter, N. Oswald, R. Bucher, Helv. Chim. Acta 58, 671 (1975)

[44] E. Gamp, Esr-untersuchungen über den jahn-teller-effekt in oktaedrischen kupfer (ii)-komplexen mit trigonalen dreizähnigen liganden. Ph.D. thesis, ETH Zürich (1980)

[45] K. Raghavachari, R.C. Haddon, T.A. Miller, V.E. Bondybey, J. Chem. Phys. 79, 1387 (1983)

[46] R. Lindner, K. Müller-Dethlefs, E. Wedum, K. Haber, E.R. Grant, Science 271, 1698 (1996)

[47] K. Muller-Dethlefs, J.B. Peel, J. Chem. Phys. 111, 10550 (1999)

[48] B.E. Applegate, T.A. Miller, J. Chem. Phys. 117, 10654 (2002)

[49] H. Köppel, L.S. Cederbaum, W. Domcke, J. Chem. Phys. 89, 2023 (1988)

[50] M. Döscher, H. Köppel, P.G. Szalay, J. Chem. Phys. 117, 2645 (2002)

[51] M. Döscher, H. Köppel, I. Baldea, H.D. Meyer, P.G. Szalay, J. Chem. Phys. 117, 2657 (2002)

[52] I. Baldea, H. Köppel, J. Chem. Phys. 124, 064101 (2006)

[53] V. Perebeinos, P.B. Allen, M. Pederson, Phys. Rev. A 72, 012501 (2005)

[54] K. Tokunaga, T. Sato, K. Tanaka, J. Chem. Phys. 124, 154303 (2006)

[55] S. Sardar, A.K. Paul, R. Sharma, S. Adhikari, J. Chem. Phys. 130, 144302 (2009) 